\title{
Controlling wildlife fungal disease spread: in vitro efficacy of disinfectants against Batrachochytrium dendrobatidis and Mucor amphibiorum
}

\author{
Rebecca Webb ${ }^{1, *}$, Annie Philips ${ }^{2}$, Rick Speare ${ }^{1}$, Joanne Connolly ${ }^{3,4}$, Lee Berger ${ }^{1}$ \\ ${ }^{1}$ Amphibian Disease Ecology Group, School of Public Health, Tropical Medicine and Rehabilitation Sciences, \\ James Cook University, Townsville, Queensland 4811, Australia \\ ${ }^{2}$ Biodiversity Conservation Branch, DPIPWE, 134 Macquarie Street, Hobart, Tasmania 7000, Australia \\ ${ }^{3}$ EH Graham Centre for Agricultural Innovation, Charles Sturt University, Wagga Wagga, New South Wales 2678, Australia \\ ${ }^{4}$ School of Animal and Veterinary Sciences, Charles Sturt University, Wagga Wagga, New South Wales 2678, Australia
}

\begin{abstract}
Chytridiomycosis in amphibians, and mucormycosis in the platypus Ornithorhynchus anatinus and amphibians, are serious fungal diseases affecting these aquatic taxa. In Tasmania, Australia, the fungi that cause these diseases overlap in range along with Phytophthora cinnamomi $(P C)$, an invasive fungal plant pathogen. To identify disinfectants that may be useful to reduce anthropogenic spread of these fungi to uninfected wilderness areas, for example by bush walkers and forestry or fire-fighting operations, we tested 3 disinfectants and a fire-fighting foam against Mucor amphibiorum (Ma) and tested 1 disinfectant and the foam against Batrachochytrium dendrobatidis $(B d)$. Combining the present study with previous work we found $B d$ was more susceptible to all 4 chemicals than Ma. Phytoclean, a disinfectant used at 2 to $10 \%$ for $30 \mathrm{~s}$ to control $P_{C}$, killed cultures of $B d$ at $0.075 \%$ and $M a$ at $5 \%$, when also applied for $30 \mathrm{~s}$. The disinfectant F10sc was not effective against $M a$ at standard exposures, but previous work shows $B d$ is killed at $0.03 \%$ with a 1 min exposure. Path-X is effective against $B d$ at $0.001 \%$ with a $30 \mathrm{~s}$ exposure and killed $M a$ at $1 \%$ with a 5 min exposure. Forexpan $\mathrm{S}$, a foam added to water at 0.1 to $1 \%$ to control forest fires, killed $B d$ but not Ma when used at $1 \%$ for 2 min. Therefore, Phytoclean and Path-X have broader efficacy, although Path-X has not been trialled against $P_{C}$. Interestingly a positive mating strain of $M a$ (from a platypus) was more resistant to disinfectants than a negative strain (from a frog). Current protocols against $P_{C}$ that involve high concentrations $(10 \%)$ of Phytoclean are likely to reduce spread of pathogenic wildlife fungi, which is important for protecting biodiversity.
\end{abstract}

KEY WORDS: Chytridiomycosis - Mucormycosis - Pathogenic fungi - Platypus - Amphibian · Phytophthora cinnamomi

\section{INTRODUCTION}

Emerging diseases of wildlife are a serious threat to biodiversity (Daszak et al. 2000) and a number of fungal diseases have recently had catastrophic effects on wildlife populations. Chytridiomycosis of amphibians and mucormycosis of the platypus Ornithorhynchus anatinus and amphibians are 2 important fungal diseases that have been selected as priorities for control in Australia (Allan \& Gartenstein
2010). Phytophthora cinnamomi $\left(P_{C}\right)$ is an emerging fungal plant pathogen in native Australian forests. These 3 pathogens have infectious stages that can occur outside the host, such as in soil or pond and stream water. There is concern that these fungi may be spread by fire-fighting or forestry activities that involve transporting water and machinery. These diseases have emerged and spread recently but are not yet established in all suitable areas (e.g. Murray et al. 2011); hence, using disinfectants to reduce 
the risk of further spread is an important control measure.

Mucormycosis, caused by the zygomycete Mucor amphibiorum (Ma), is the most significant disease affecting platypus in Tasmania, although it has not been detected in mainland platypuses (Connolly et al. 1998). In the platypus, infection causes an ulcerative dermatitis, and the fungus may disseminate to internal organs (Obendorf et al. 1993). Infected platypuses can develop impaired thermoregulation and mobility and may die. Mucormycosis also occurs in mainland Australian amphibians but has not been detected in Tasmanian frogs (Connolly et al. 1998). It can cause a fatal, systemic infection in captive and wild frogs and cane toads Bufo marinus (Berger et al. 1997, Speare et al. 1997, Creeper et al. 1998). A platypus strain of the fungus was able to experimentally infect amphibians (Stewart \& Munday 2005). Ma is heterophallic; positive strains produce resistant zygospores if mated with a negative strain, but only positive strains have been found in Tasmania (Schipper 1978, Connolly 2009). Ma also reproduces asexually via infective sporangiospores (Stewart \& Munday 2005). Infection experiments in toads suggest that the positive strains of Ma are more pathogenic. It grows on soil and may largely be a saprobic organism that opportunistically infects animals (Speare et al. 1994). It has been speculated that the spread of mucormycosis around Tasmania was caused in part by human activities (Gust \& Griffiths 2009). Mucormycosis was initially discovered in Elizabeth River, central-eastern Tasmania, in 1982. Since then it appears to have spread across northern Tasmania and has been found in 11 river catchments, or about $24 \%$ of the state (Gust \& Griffiths 2009).

Chytridiomycosis, caused by the chytridiomycete Batrachochytrium dendrobatidis $(B d)$, is an invasive disease that has caused amphibian declines worldwide (Skerratt et al. 2007). There are 2 main stages in the life cycle: the motile, waterborne and infective zoospore, and the zoosporangium that grows within epidermal cells. A likely mechanism of spread is via natural and anthropogenic movement of infected amphibians, but movement via water and moist soil is also possible (Berger et al. 2009). Saprobic growth is suspected but has not been proven. In Tasmania the large southwestern World Heritage Area, which contains 3 endemic frog species (Driessen \& Mallick 2003), appears to be largely free from $B d$, while it occurs throughout much of the rest of the state (Obendorf \& Dalton 2006, Pauza et al. 2010).

In Australia, the introduced plant disease 'Phytophthora root rot' or 'wildflower die-back' caused by the zoosporic oomycete $P_{C}$, is widespread and is threatening a number of plant species and communities (Rudman 2005). It is of special concern in Tasmanian heathlands, dry forests and buttongrass moorlands. Though primarily a soil-inhabiting pathogen, $P_{C}$ can also contaminate creeks and water bodies.

There is concern that these 3 diseases could be further spread around the state anthropogenically such as by tourists, forestry machinery or fire-fighting operations. To prevent further spread of $P_{C}$ within Tasmania, the Department of Primary Industries, Parks, Water and Environment has developed guidelines that involve washing machinery, vehicles, hiking boots and equipment. Where risks are significant the additional use of the disinfectant Phytoclean (Boldscape) is recommended. Phytoclean (active ingredient: benzalkonium chloride, $30 \% \mathrm{w} / \mathrm{w}$ ) is designed for the control of $P_{C}$ in earth-moving, quarrying, horticultural and plantation industries. In this study we tested the effectiveness of Phytoclean against $B d$ and Ma to check whether current guidelines designed to minimise the spread of root rot are simultaneously reducing the spread of these wildlife pathogens.

Effective disinfectants for $B d$ have been identified for use in laboratories, in captive husbandry and on field equipment to reduce human-mediated spread (Johnson et al. 2003, Webb et al. 2007, Phillott et al. 2010). Two disinfectants were effective at low concentrations: F10 super concentrate disinfectant 'F10sc' (active ingredients: benzalkonium chloride, $5.4 \% \mathrm{w} / \mathrm{w}$, and polyhexamethylene biguanide hydrochloride, $0.4 \%$ w/w; Health and Hygiene) and Path-X (active ingredient: didecyl dimethyl ammonium chloride, $12 \%$ w/w; Nutri-Tech Solutions) (Johnson et al. 2003, Webb et al. 2007). Hence, we tested the effectiveness of F10sc and Path-X against Ma.

We also tested Forexpan S (Angus Fire) for disinfectant activity against $M a$ and $B d$. Forexpan $\mathrm{S}$ is a synthetic fire-fighting foam concentrate, added to water to control forestry or wildland fires. It contains hydrocarbon surfactants, glycol solvent and a foam stabiliser and reduces surface tension, which allows water to penetrate into burning material (Angus Fire website: www.angusfire.co.uk, accessed 2009). As water taken into fire trucks may be sourced from any convenient water body, fire fighters may unintentionally be spreading aquatic pathogens.

\section{MATERIALS AND METHODS}

Determining the minimum lethal concentration (MLC) of a disinfectant is necessary to develop disin- 
fection protocols. The MLC is the lowest concentration that will prevent the growth of an organism after subculture onto media free from the antimicrobial; it describes fungicidal effects. Owing to the differences in growth form between $B d$ and $M a$, different methods were used for fungicidal tests to determine the MLC. The methods (Methods 1 and 2) were similar in that the fungal culture and supporting structure were exposed to a solution of the test compound then rinsed, returned to culture media, incubated and monitored for growth. Initially we used concentrations recommended by the manufacturer and if these were effective, concentrations were decreased until cultures survived (see Table 1 for concentration and exposure ranges tested). The minimum inhibitory concentration (MIC) is the lowest concentration that will inhibit the visible growth of a microorganism; it describes fungistatic effects and provides further information on antimicrobial action (Quinn et al. 2002). Method 3 was used to determine the minimum inhibitory concentration (MIC) of Phytoclean for both fungi.

\section{Method 1. Fungicidal effects on Batrachochytrium dendrobatidis}

Fungicidal effects of compounds on $B d$ were tested by using a published method (Johnson et al. 2003, Webb et al. 2007). An active culture of an isolate from Queensland, 'Tully-Lrheocola-06-LB-1' (Berger et al. 2005), was grown as a monolayer in 96-well plates (Techo Plastic Products) with tryptone, gelatin hydrolysate, lactose (TGhL) broth (Sigma and Oxoid) for $4 \mathrm{~d}$, then exposed to the test product at various concentrations and exposure times (see Table 1) with 8 replicate wells. Phytoclean and fire-foam were diluted with sterile water. Positive growth controls were treated with water only and remained alive during the experiment. After exposure the test product was removed, and wells were rinsed with TGhL broth, filled with fresh TGhL broth and incubated at $23^{\circ} \mathrm{C}$. The plates were checked daily for $1 \mathrm{wk}$ to detect the presence of motile zoospores, which indicated culture survival. A $100 \%$ kill of all 8 replicates was required for the product to be considered effective.

\section{Method 2. Fungicidal effects on Mucor amphibiorum}

Fungicidal activities of compounds on $M a$ were tested by using a method similar to 'Protocol 2' described by Alderman (1982), except that the fungi was grown on glass coverslips rather than filter membranes to enable the fungus to be dipped into the disinfectant. This method is similar to routinely used 'carrier tests' (Swart \& Hilgren 2001). Two isolates of Ma were obtained from culture collections: isolate '40.41', a positive mating strain isolated from an ulcer on a Tasmanian platypus, and '40.50', a negative mating strain, isolated in 1977 from a captive frog (Dendrobates sp.) in Germany. Ma was grown on Sabouraud's dextrose agar (SDA) (Oxoid) for several days at $25^{\circ} \mathrm{C}$. Square pieces (approximately $2 \mathrm{~cm}^{2}$ ) of fungi and agar were cut and placed on new SDA with fungus side up and covered with sterile $40 \times 24 \mathrm{~mm}$ glass coverslips (Livingstone). Further squares of fungi were inverted and placed on top of the coverslips. These culture plates were then sealed and incubated at $25^{\circ} \mathrm{C}$ for $4 \mathrm{~d}$. Hyphae grew on both sides of the coverslips and remained attached when the agar squares were peeled off.

The coverslips with attached hyphae and sporangiospores were exposed to disinfectants in individual, sterile, $70 \mathrm{ml}$ specimen containers (Sarstedt) containing $50 \mathrm{ml}$ of disinfectant or fire-foam solution (see Table 1 for concentrations). Coverslips were submerged in the liquid by using sterile forceps. After the exposure period, the coverslip was removed by using a new pair of sterile forceps, shaken to remove excess liquid, submerged in sterile water for $10 \mathrm{~s}$ and shaken again before being placed on a clean SDA plate. The plates were sealed with Parafilm (Pechiney Plastic Packaging) and incubated at $25^{\circ} \mathrm{C}$. Plates were checked daily for a week for fungal growth around the coverslip. Surviving fungi quickly colonised the surrounding agar indicating that the disinfectant exposure was ineffective. Each experiment included a positive control treatment of sterile water. A negative control treatment of $70 \%$ ethanol was also included in each experiment to check for contamination by airborne sporangiospores. The results were only considered valid if the positive controls showed growth and the negative controls did not. Each experiment had 2 replicates for a particular concentration/ exposure combination, and if the culture was killed then the test was repeated at least 3 times on different days. The MLC was the lowest concentration that killed all replicates of both Ma strains.

\section{Method 3. Fungistatic effects on Batrachochytrium dendrobatidis and Mucor amphibiorum}

Method 3 was aimed at determining the MIC of Phytoclean, since it appeared to be a promising 
broad spectrum disinfectant. This method was used on both fungi, although $B d$ was grown on TGhL agar and Ma was grown on SDA. Method 3 is similar to 'Protocol 1' described by Alderman (1982). Agar was autoclaved at $121^{\circ} \mathrm{C}$ for $15 \mathrm{~min}$ then allowed to cool for a few hours in hot water. Phytoclean was added to the warm liquid agar to produce various concentrations (ranging from 10\% down to $0.001 \%$ ). Positive growth control plates contained no Phytoclean. Cultures of $B d$ and both mating types of Ma were grown on TGhL and SDA agar at $23^{\circ} \mathrm{C}$ and $25^{\circ} \mathrm{C}$, respectively. After $3 \mathrm{~d}, 1 \mathrm{~cm}^{2}$ pieces were cut from the growing edge of the cultures and placed, fungus side down, onto the agar containing Phytoclean. Plates were checked daily for $1 \mathrm{wk}$ and each test was replicated 3 times. Uninhibited fungi colonised the surrounding agar within a few days. The MIC was the lowest concentration without growth.

\section{RESULTS \\ Phytoclean}

With a 30 s exposure, Phytoclean was effective against $B d$ at only $0.075 \%$, whereas $5 \%$ was required to kill Ma (Tables 1 \& 2). However, the negative $M a$ strain 40.50 was more sensitive than the positive strain 40.41 and was killed by $4 \%$ Phytoclean within $30 \mathrm{~s}$.

The MIC testing (Method 3) showed 0.005\% $\left(0.05 \mathrm{ml} \mathrm{l}^{-1}\right)$ Phytoclean inhibited growth of $B d$ and $0.08 \%\left(0.8 \mathrm{ml} \mathrm{l}^{-1}\right)$ inhibited growth of $\mathrm{Ma}$.

\section{Forexpan S}

$B d$ was killed by $1 \%$ Forexpan $\mathrm{S}$ if the exposure time was at least $2 \mathrm{~min}$. Ma was not killed by the maximum concentration and exposure combination tested, $1 \%$ for 5 min. Higher concentrations were not tested as they are not applicable.

\section{F10sc}

The positive mating strain of Ma 40.41 was more resistant to F10sc than the negative strain 40.50 . F10sc used at $5 \%$ for 10 min was effective against the negative strain, but only killed $40 \%$ of positive strain replicates (Table 1). To kill fungal spores the manufacturers recommend F10sc is used at concentrations
Table 1. Testing schedule for disinifectants used against in vitro cultures of Batrachochytrium dendrobatidis $(B d)$ and Mucor amphibiorum (Ma) in the present study. Concentrations refer to diluted commercial disinfectant, not active ingredients. Efficacy = replicates killed / replicates tested; percentage killed is indicated in parentheses

\begin{tabular}{|c|c|c|c|}
\hline $\begin{array}{l}\text { Disinfectant and } \\
\text { target species }\end{array}$ & $\begin{array}{l}\text { Conc. } \\
(\%)\end{array}$ & $\begin{array}{l}\text { Exposure } \\
\text { time (min) }\end{array}$ & $\begin{array}{l}\text { Efficacy } \\
\text { (\% killed) }\end{array}$ \\
\hline \multicolumn{4}{|l|}{ Phytoclean } \\
\hline \multirow[t]{7}{*}{$B d$} & 0.01 & $0.5,1,5$ & $0 / 8(0)$ \\
\hline & 0.03 & $0.5,1$ & $0 / 8(0)$ \\
\hline & 0.03 & 5 & $8 / 8(100)$ \\
\hline & 0.05 & 0.5 & $0 / 8(0)$ \\
\hline & 0.05 & 1,5 & 8/8 (100) \\
\hline & 0.08 & $0.5,1,5$ & 8/8 (100) \\
\hline & 0.1 & $0.5,1,5$ & 8/8 (100) \\
\hline$M a_{1}$ +ve strain & 2 & 5 & $7 / 8(87.5)$ \\
\hline \multirow[t]{2}{*}{40.41} & 4 & 0.5 & $1 / 4(25)$ \\
\hline & 5 & 0.5 & $8 / 8(100)$ \\
\hline$M a_{1}$-ve strain & 2 & 5 & $8 / 8(100)$ \\
\hline \multirow[t]{2}{*}{40.50} & 4 & 0.5 & $4 / 4(100)$ \\
\hline & 5 & 0.5 & $6 / 6(100)$ \\
\hline \multicolumn{4}{|l|}{ Forexpan S } \\
\hline \multirow[t]{4}{*}{$B d$} & 0.10 & $0.5,1,5$ & $0 / 8(0)$ \\
\hline & 0.50 & $0.5,1,5$ & $0 / 8(0)$ \\
\hline & 1 & 0.5 & $0 / 8(0)$ \\
\hline & 1 & 1,5 & $8 / 8(100)$ \\
\hline$M a_{1}+$ ve strain & 0.10 & 0.5 & $0 / 2(0)$ \\
\hline 40.41 & 1 & $0.5,5$ & $0 / 2(0)$ \\
\hline$M a_{1}$-ve strain & 0.10 & 0.5 & $0 / 2(0)$ \\
\hline 40.50 & 1 & $0.5,5$ & $0 / 2(0)$ \\
\hline \multicolumn{4}{|l|}{ F10sc } \\
\hline$B d$ & \multicolumn{3}{|c|}{ Not tested here; see Webb et al. (2007 } \\
\hline$M a_{1}$ +ve strain & 0.8 & 30 & $0 / 6(0)$ \\
\hline \multirow[t]{2}{*}{40.41} & 5 & 10 & $2 / 5(40)$ \\
\hline & 5 & 20 & $5 / 6(83)$ \\
\hline$M a_{1}$-ve strain & 0.8 & 30 & $5 / 6(83)$ \\
\hline \multirow[t]{3}{*}{40.50} & 5 & 5 & $1 / 4(25)$ \\
\hline & 5 & 10 & 9/9 (100) \\
\hline & 5 & 20 & $6 / 6(100)$ \\
\hline \multicolumn{4}{|l|}{ Path-X } \\
\hline$B d \quad \mathrm{~N}$ & \multicolumn{3}{|c|}{ Not tested here; see Johnson et al. (2003) } \\
\hline$M a,+$ ve strain & 0.5 & 10 & 4/6 (67) \\
\hline 40.41 & 1 & 5,10 & $6 / 6(100)$ \\
\hline$M a_{1}$-ve strain & 0.5 & 10 & 6/6 (100) \\
\hline 40.50 & 1 & 5,10 & 6/6 (100) \\
\hline
\end{tabular}

between 0.4 and $0.8 \%$ for $30 \mathrm{~min}$, which was ineffective against $M a$.

\section{Path-X}

Path-X at $1 \%$ for 5 min was effective against $M a$ and killed the fungus in all 6 replicates tested. Path$\mathrm{X}$ at $0.5 \%$ for $10 \mathrm{~min}$ only killed the negative strain. 
Table 2. Summary of disinfectant protocols effective against cultured Batrachochytrium dendrobatidis $(B d)$ and Mucor amphibiorum (Ma), using data from the present study and from previous work. Higher exposure concentrations or times should be used in applied situations. Concentrations refer to dilutions of the commercial compounds, not active ingredients. MLC: minimum lethal concentration; ne: not effective $(<100 \%$ efficacy at maximum concentrations and times tested; see Table 1)

\begin{tabular}{|c|c|c|c|c|c|c|}
\hline \multirow{3}{*}{ Disinfectant } & \multicolumn{3}{|c|}{$-B d$} & \multicolumn{2}{|r|}{ - Ma } & \multirow{3}{*}{$\begin{array}{c}\text { Exposure } \\
\text { time } \\
\text { (min) }\end{array}$} \\
\hline & \multicolumn{2}{|c|}{ MLC } & \multirow{2}{*}{$\begin{array}{c}\text { Exposure } \\
\text { time } \\
\text { (min) }\end{array}$} & \multicolumn{2}{|c|}{ MLC } & \\
\hline & $(\%)$ & $\left(\mathrm{ml} \mathrm{l}^{-1}\right)$ & & $(\%)$ & $\left(\mathrm{ml} \mathrm{l}^{-1}\right)$ & \\
\hline Phytoclean & 0.075 & 0.75 & 0.5 & 5 & 50 & 0.5 \\
\hline Forexpan S & 1 & 10 & 2 & ne & & \\
\hline F10sc & $0.033^{\mathrm{a}}$ & 0.33 & $1^{\mathrm{a}}$ & ne & & \\
\hline \multirow[t]{2}{*}{ Path-X } & $0.001^{\mathrm{b}}$ & 0.01 & $0.5^{\mathrm{b}}$ & 1 & 10 & 5 \\
\hline & $0.0001^{\mathrm{b}}$ & 0.001 & $2^{\mathrm{b}}$ & & & \\
\hline
\end{tabular}

\section{DISCUSSION}

The disinfectant Phytoclean was effective against $B d$ but not against $M a$ at the lower concentrations used widely for control of $P_{C}$. The manufacturers recommend $2 \%$ Phytoclean is used for $>30 \mathrm{~s}$ when washing down machinery and cleaning benches and tools, and recommend 10\% Phytoclean for foot baths (Phytoclean website: www.phytoclean.com.au/, accessed 2009). These concentrations are both well above the level effective against $B d$ as $0.075 \%$ will kill sporangia within $30 \mathrm{~s}$. However, the lower recommended exposure $(2 \%$ for $30 \mathrm{~s})$ is not effective against cultures of $M a$, which required at least $5 \%$ for $30 \mathrm{~s}$. If standard recommendations for footbaths (10\% Phytoclean) are followed, all 3 pathogens will be killed. Phytoclean is already in use in Tasmania, particularly for Phytophthora control in conservation areas. It is non-corrosive and there have been no reports of it degrading or damaging equipment (T. Rudman pers. comm.).

Forexpan $\mathrm{S}$ fire foam has only minor potential for controlling $B d$. Forexpan $\mathrm{S}$ at a concentration of $1 \%$ for at least 1 min killed $B d$, but $M a$ was not affected by $1 \%$ Forexpan $\mathrm{S}$, even after $5 \mathrm{~min}$. The fire foam is recommended to be used at between 0.1 and $1 \%$, and is typically added to the water once spraying commences (A. Pyrke, DPIPWE, Hobart, pers. comm.). Therefore, it has a short exposure time $(<30 \mathrm{~s})$ before the water enters the environment. However, if a helicopter is used to fight fires, the exposure time was estimated at $5 \mathrm{~min}$, which would kill $B d$ if a consistent concentration of $1 \%$ was achieved. The high temperatures associated with a bush fire would be expected to kill both fungi, particularly $B d$, which dies at $37^{\circ} \mathrm{C}$ within $4 \mathrm{~h}$ (Johnson et al. 2003). However, while water landing on the fire would be sterilised, there is concern regarding run-off of cold water, which highlights the need for investigation into fungicides to add to water used for fire fighting.

F10sc is an effective disinfectant against $B d$ at concentrations as low as $0.033 \%$ for $1 \mathrm{~min}$ (Webb et al. 2007). Unfortunately F10sc was not as effective against $M a$, and the manufacturer's lengthy recommended protocols did not completely kill both $M a$ strains. To determine the MLC for Ma requires further work.

Path-X is an agricultural disinfectant and the active ingredient is a quaternary ammonium compound. Path- $\mathrm{X}$ is recommended to be used at a concentration of $1 \%$ for at least 10 min for general disinfection of equipment and hands and for use in foot baths. This protocol is effective against $M a$ and $B d$ (Webb et al. 2007), which were killed at concentrations of 1 and $0.0001 \%$, respectively. Path-X has not yet been tested against $P_{C}$, but it is likely to be effective since other quaternary ammonium compounds were shown to inhibit $P C$ at low exposures (Noske \& Shearer 1985).

Table 2 summarizes disinfectant protocols effective against cultured $B d$ and $M a$, using data from the present study and previous work. At present, Phytoclean appears to be the best choice as it is known to be effective against all 3 fungi, is already used widely, works rapidly and is non-corrosive (Material Safety Data Sheet No. 24-7755, Chemwatch CD 2010/1). Currently established protocols for $P_{C}$ also kill $B d$, but for them to also be effective against $M a$ requires increasing the time required for the washdown and instrument cleaning. As $5 \%$ Phytoclean for $30 \mathrm{~s}$ killed cultures of $M a$, we suggest applying $5 \%$ Phytoclean for $1 \mathrm{~min}$ or $10 \%$ for $30 \mathrm{~s}$.

The MIC and MLC in this study refer to in vitro testing, using pure fungal cultures and sterile materials. As soil and organic matter can interfere with disinfection, higher concentrations and exposure times should be used in the field, after first washing equipment to remove dirt. $B d$ is difficult to culture from non-sterile substrates, so testing its survival in real-world situations, such as on used equipment, is problematic.

All 3 disinfectants tested here are human irritants and as they are toxic to aquatic organisms disposal 
into the environment, especially water bodies, should be avoided. Disinfectants can be applied to equipment and then rinsed to reduce environmental contamination. Further work should compare sublethal effects of low concentrations on tadpoles and other aquatic organisms. For example Schmidt et al. (2009) showed that bleach is highly toxic to tadpoles and zooplankton and recommended Virkon $\mathrm{S}$ as a safer disinfectant for field use. As Virkon $\mathrm{S}$ is effective against $B d$ (Johnson et al. 2003), it could also be trialled against $M a$ and $P C$.

The susceptibility of $M a$ to chemical disinfection differed between the strains. The negative mating strain 40.50 was more susceptible to Phytoclean, F10sc and Path-X. Infection experiments using toads have suggested that the positive strains of $M a$ are also more pathogenic and cause more severe infections. The reason may be that positive strains produce more spherules containing daughter cells (Stewart \& Munday 2005). The positive mating type of a plant pathogen, Mucor piriformis, was also more pathogenic and caused larger lesions than did the negative mating type (Michailides \& Spotts 1986). Another reason for differences in susceptibility between the strains of Ma could be related to their origin (amphibian versus platypus), which is associated with genetic differences (Connolly et al. 2010).

Our sensitivity trials for Ma only tested the actively growing hyphae and sporangiospores (asexual reproduction). Zygospores (sexual reproduction) are assumed to be absent from Tasmania as only the positive mating strain has been isolated, despite numerous culture attempts from samples including platypus ulcers, frog faeces, soil, water and ticks (Connolly et al. 1998, Stewart \& Munday 2005). However, both strains do occur in mainland Australia so further work should test the resistance of zygospores to chemical disinfectants.

\section{CONCLUSION}

From this study and previous work (Johnson et al. 2003, Webb et al. 2007), we conclude Phytoclean and Path-X are highly effective against $B d$ and moderate levels kill Ma. F10sc was effective against $B d$ but not Ma when the manufacturer's directions were followed. Forexpan S only killed $B d$ when the exposure was higher than is typically used for fire fighting. Out of the 4 compounds tested, Phytoclean appears the most useful as it acts rapidly, and current protocols for $P_{C}$ are effective against $B d$ and could be modified to be effective against Ma. In future, Path-X could be tested against $P_{C}$ in case this is an alternative disinfectant for all 3 fungi. This knowledge is key to developing hygiene protocols that will reduce the risk of spreading pathogens to new wilderness areas, for example in washdown stations for bush walkers, field biologists and forestry or fire-fighting operations.

Acknowledgements. We thank K. Weeks for kindly sending Ma cultures and A. Thomas for help with identifying Ma. We are grateful to K. Henning from Nutri-Tech and to A. Pyrke for providing antifungal compounds. The EH Graham Centre for Agricultural Innovation is jointly supported by Charles Sturt University and the NSW Department of Primary Industries. This work was supported by the Australian Department of Environment and Heritage and the Tasmanian Department of Primary Industries, Parks, Water and Environment.

\section{LITERATURE CITED}

Alderman DJ (1982) In vitro testing of fisheries chemotherapeutants. J Fish Dis 5:113-123

Allan K, Gartenstein S (2010) Keeping it clean: a Tasmanian field hygiene manual to prevent the spread of freshwater pests and pathogens. NRM South, Hobart. www.dpiw. tas.gov.au/internnsf/Publications/LBUN-8896DT?open

Berger L, Speare R, Humphrey J (1997) Mucormycosis in a free-ranging green tree frog from Australia. J Wildl Dis 33:903-907

Berger L, Marantelli G, Skerratt LF, Speare R (2005) Virulence of the amphibian chytrid fungus Batrachochytrium dendrobatidis varies with the strain. Dis Aquat Org 68: 47-50

Berger L, Longcore J, Speare R, Hyatt A, Skerratt L (2009) Fungal diseases in amphibians. In: Heatwole H, Wilkinson J (eds) Amphibian decline: disease, parasites, maladies and pollution. Amphibian biology, Vol 8. Surrey Beatty \& Sons, Chipping Norton, p 2986-3052

Connolly JH (2009) A review of mucormycosis in the platypus (Ornithorhynchus anatinus). J Zool 57:235-244

Connolly JH, Obendorf DL, Whittington RJ, Muir DB (1998) Causes of morbidity and mortality in platypus (Ornithorhynchus anatinus) from Tasmania, with particular reference to Mucor amphibiorum infection. Aust Mammal 20: $177-187$

- Connolly JH, Stodart B, Ash G (2010) Genotypic analysis of Mucor from the platypus and amphibian in Australia. J Wildl Dis 46:55-69

Creeper JH, Main DC, Berger L, Huntress S, Boardman W (1998) An outbreak of mucormycosis in slender tree frogs (Litoria adelensis) and white-lipped tree frogs (Litoria infrafrenata). Aust Vet J 76:761-762

> Daszak P, Cunningham AA, Hyatt AD (2000) Emerging infectious diseases of wildlife- threats to biodiversity and human health. Science 287:443-449

Driessen MM, Mallick SA (2003) The vertebrate fauna of the Tasmanian Wilderness World Heritage Area. Pac Conserv Biol 9:187-206

Gust N, Griffiths J (2009) Platypus mucormycosis and its conservation implications. Australas Mycol 28:1-8

Johnson ML, Berger L, Philips L, Speare R (2003) Fungicidal effects of chemical disinfectants, UV light, desiccation 
and heat on the amphibian chytrid Batrachochytrium dendrobatidis. Dis Aquat Org 57:255-260

Michailides TJ, Spotts RA (1986) Mating types of Mucor piriformis isolated from soil and pear fruit in Oregon orchards. Mycologia 78:766-770

Murray KA, Retallick RWR, Puschendorf R, Skerratt LF and others (2011) Assessing spatial patterns of disease risk to biodiversity: implications for the management of the amphibian pathogen, Batrachochytrium dendrobatidis. J Appl Ecol 48:163-173

Noske GL, Shearer BL (1985) Quaternary ammonium compounds were more effective than a phenolic compound or sodium hypochlorite in inhibiting growth of Phytophthora cinnamoni (Rands). Australas Plant Pathol 14: $37-40$

Obendorf D, Dalton A (2006) A survey for the presence of the amphibian chytrid fungus (Batrachochytrium dendrobatidis) in Tasmania. Pap Proc R Soc Tasman 140: 25-29

Obendorf DL, Peel BF, Munday BL (1993) Mucor amphibiorum infection in platypus (Ornithorhyncus anatinus) from Tasmania. J Wildl Dis 29:485-487

Pauza MD, Driessen MM, Skerratt LF (2010) Distribution and risk factors for spread of amphibian chytrid fungus Batrachochytrium dendrobatidis in the Tasmanian Wilderness World Heritage Area. Dis Aquat Org 92: 193-199

Phillott AD, Speare R, Hines HB, Meyer E and others (2010) Minimising exposure of amphibians to pathogens during field studies. Dis Aquat Org 92:175-185

Quinn PJ, Markey BK, Carter ME, Donnelly WJ, Leonard FC (2002) Veterinary microbiology and microbial disease. Blackwell Science, Ames, IA

Editorial responsibility: David Marcogliese, Montreal, Quebec, Canada
Rudman T (2005) Interim Phytophthora cinnamomi management guidelines. Nature Conservation Report 05/07. Biodiversity Conservation Branch, Department of Primary Industries, Parks, Water and Environment, Hobart

Schipper MAA (1978) On certain species of Mucor with a key to all accepted species. Stud Mycol 17:1-52

Schmidt BR, Geiser C, Peyer N, Keller N, Rutte M (2009) Assessing whether disinfectants against the fungus $B a-$ trachochytrium dendrobatidis have negative effects on tadpoles and zooplankton. Amphib-Reptilia 30:313-319

Skerratt LF, Berger L, Speare R, Cashins S and others (2007) The spread of chytridiomycosis has caused the rapid global decline and extinction of frogs. EcoHealth 4: 125-134

Speare R, Thomas AD, O'Shea P, Shipton WA (1994) Mucor amphibiorum in the cane toad, Bufo marinus, in Australia. J Wildl Dis 30:399-407

Speare R, Berger L, O'Shea P, Ladds PW, Thomas AD (1997) Pathology of mucormycosis of cane toads in Australia. J Wildl Dis 33:105-111

Stewart NJ, Munday BL (2005) Possible differences in pathogenicity between cane toad-, frog- and platypusderived isolates of Mucor amphibiorum, and a platypusderived isolate of Mucor circinelloides. Med Mycol 43: $127-132$

Swart SK, Hilgren JD (2001) Methods of testing fungicides. In: Block $\mathrm{S}$ (ed) Disinfection, sterilization and preservation, 5th edn. Lippincott, Williams \& Wilkins, London, p 1383-1390

> Webb R, Mendez D, Berger L, Speare R (2007) Additional disinfectants effective against the amphibian chytrid fungus Batrachochytrium dendrobatidis. Dis Aquat Org 74:13-16

Submitted: March 3, 2011; Accepted: March 7, 2012 Proofs received from author(s): May 21, 2012 